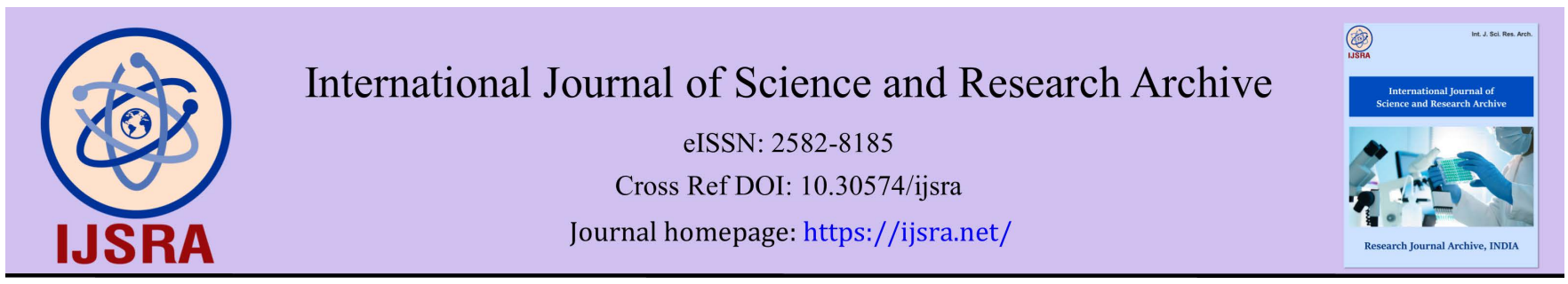

(RESEARCH ARTICLE)

\title{
The effect of intense physical activity session with glutamine supplementation on selected factors of wrestlers' immune system
}

\author{
Sepehri Manesh Mohammad ${ }^{1, *}$, Abbasi Moghadam Mahdi ${ }^{1}$ and Sohrabi Parisa ${ }^{2}$ \\ ${ }^{1}$ Khorramabad Technical and Vocational College, Khorramabad, Lorestan, Iran. \\ ${ }^{2}$ Shahid Rahimi Hospital, Lorestan University of Medical Sciences, Khorramabad, Lorestan, Iran.
}

International Journal of Science and Research Archive, 2021, 02(02), 145-150

Publication history: Received on 13 April 2021; revised on 16 May 2021; accepted on 20 May 2021

Article DOI: https://doi.org/10.30574/ijsra.2021.2.2.0070

\begin{abstract}
Athletes with heavy training loads are prone to infectious diseases. It has been shown that these exercises may suppress immune function. The aim of this study was to investigate the effect of one session of intense physical activity with glutamine supplementation on selected factors of wrestlers' immune system. For this purpose, 20 wrestlers were selected and randomly divided into two groups of supplement and placebo. The first blood sample was taken from the subjects. Then they did the Bruce test. Immediately after the end of the test, blood samples were taken from the subjects for the second time and then to the supplement group. The glutamine supplement was given the same amount of maltodextrin per kilogram of body weight and the placebo group. Blood samples were taken from the subjects for the third and fourth time 1 and 2.5 hours after taking the supplement or placebo. After measuring blood factors, the findings were statistically analyzed (ANOVA) at a significance level of $p<0.05$. The results showed that the percentage of neutrophils in the supplement group increased significantly 2.5 hours after supplementation compared to immediately after exercise and 1 hour after supplementation. This increase was less in the supplement group than in the placebo group. The percentage of lymphocytes in the supplement group decreased significantly 2.5 hours after supplementation compared to the pre-test, immediately after exercise and 1 hour after supplementation, which was less in the supplement group than the placebo group. The percentage of monocytes in the supplement group increased significantly 2.5 hours after supplementation compared to immediately after exercise. This increase was less in the supplement group than in the placebo group. The results of this study showed that glutamine supplementation during strenuous exercise has beneficial effects on the immune system of athletes.
\end{abstract}

Keywords: Glutamine; Immune system; Intense physical activity

\section{Introduction}

The function of the human body is affected by different systems. Exercise activities of varying intensity and duration cause a variety of responses in these systems. The immune system is one of the systems that undergoes major changes as a result of sports activities. The most important function of the immune system is to protect the body against infectious diseases [1]. Evidence shows, while moderate-intensity physical activity has beneficial effects on the immune system, intense exercise reduces the function of the immune system [2]. Also, studies show that during intense exercise, the incidence of infectious diseases, especially upper respiratory tract infections, increases [3]. Related mechanisms include neuro-hormonal factors such as epinephrine, norepinephrine, growth hormone, cortisol, and beta-endorphins. Changes in metabolic factors during exercise, such as decreased plasma glutamine, can play an important role. Research shows that lymphocyte density decreases during strenuous exercise [4].

\footnotetext{
${ }^{*}$ Corresponding author: Mohammad Sepehri Manesh

Khorramabad Technical and Vocational College, Khorramabad, Iran.
} 
Plasma glutamine levels are important for immune function because glutamine storage is essential for the full availability of plasma leukocytes [5]. In neutrophils and macrophages, glutamine is very important for rapid energy supply [6]. Glutamine is a natural non-essential amino acid. This substance is important as a component of protein and a means of nitrogen transfer between tissues. It is used in rapidly dividing cells such as lymphocytes and neutrophils [7]. It is also important in regulating basal acid, gluconeogenesis and as an antioxidant glutathione. Glutamine is the most abundant free amino acid in human muscle and plasma. In adults, normal plasma glutamine concentrations are $550-750 \mu \mathrm{mol} / \mathrm{L}$, and skeletal muscle glutamine concentrations are $20 \mathrm{mmol} / \mathrm{L} / \mathrm{kg}$ muscle fresh weight [8]. Evidence suggests that strenuous exercise may reduce glutamine release from skeletal muscle or increase glutamine uptake by other organs or tissues that use glutamine (e.g., liver and kidney), thus limiting access. Glutamine enters the cells of the immune system [9]. The effect of acute exercise on plasma glutamine concentration is highly dependent on the duration and intensity of exercise [10]. In rats, glutamine supplementation improves neutrophil function [11, 12]. In humans, studies in patients [13] and healthy athletic volunteers [14] also confirm support for the role of plasma glutamine in immune function. In judo athletes, glutamine supplementation improved neutrophil function after a training session by increasing the oxidative pathway [14]. However, the effectiveness of glutamine supplementation in athletes is controversial because some studies have failed to demonstrate positive effects on exercise-related immunosuppression $[15,16]$.

It has been suggested that differences in the literature may be related to the rate of plasma glutamine depletion. Significant reductions in plasma glutamine (e.g., values less than $400 \mathrm{mmol} / \mathrm{L}$ ) appear to be beyond the threshold for immune dysfunction [17]. In such cases, glutamine supplementation may be beneficial for the immune system. In contrast, when plasma glutamine is only slightly reduced, glutamine supplementation appears to have no effect on immunosuppression associated with plasma glutamine depletion, and therefore, supplements will not be helpful [15 and 16].

The effect of exercise on immune system function and the interaction of infectious diseases on athletes' athletic performance are among the topics that have been considered in recent years. Evidence shows that strenuous exercise weakens the immune system and decreases plasma glutamine levels. If lowering plasma glutamine levels is effective in reducing immune function, lowering plasma glutamine by taking oral glutamine supplements should prevent damage to the immune system. However, several interventions in the use of glutamine in humans have shown that glutamine supplementation before and after exercise does not have a significant effect on changes in immune cell function, with conflicting results. The aim of the present study was to investigate the effect of glutamine supplementation on selected immune system factors of wrestlers following a session of intense physical activity.

\section{Material and methods}

\subsection{Participants}

Fifty-eight male wrestlers volunteered for the study. After eligibility assessment, 20 athletes were randomly selected to receive glutamine and placebo. Inclusion criteria were: (a) age 20 to 30 years, (b) a minimum training period of 10 hours per week, (c) at least 6 years of experience in wrestling, and (b) exclusion criteria before investigating these cases: (a) tobacco use; (B) the use of drugs that affect the immune system in the last 2 months; (C) the presence of infectious diseases diagnosed in the last two months; (D) the presence of symptoms indicating infection and disease; (e) the use of nutritional supplements containing glutamine or other chemicals that may alter immune function. Also, prior to participation, all athletes completed informed consent. Subjects did not engage in any strenuous physical activity for 48 hours before the first blood sample was taken.

\subsection{Sampling and examination}

The first blood sample was taken from the subjects and then the height and weight of the subjects were measured using a caliper and laboratory scales. Subjects then worked on the treadmill to the point of exhaustion during the Bruce test. In the second stage, blood sampling was performed after the activity. Immediately to the experimental group of glutamine supplementation at the rate of $4 \mathrm{~g}$ per $500 \mathrm{~kg}$ of body weight was given in $500 \mathrm{ml}$ of water and the control group was given the same amount of placebo (maltodextrin) and then all subjects rested and 1.5 and 2.5 hours after taking the supplement or placebo for once. Blood samples were taken from the third and fourth subjects.

\subsection{Data analysis}

Analysis of variance (ANOVA) was used to analyze the data and then Tukey post hoc test was used if there was a significant difference between the means at different stages. The level of statistical significance was considered $\mathrm{p}<0.05$. 


\section{Results}

Table 1 shows a comparison of the characteristics of the subjects, including height, weight, maximum oxygen consumption, and age, divided into two groups: placebo and supplement. All values are expressed based on the mean and standard deviation.

Table 1 Description of scientific information (criterion standard deviation).

\begin{tabular}{|l|l|l|l|l|}
\hline Group & Age & Weight & Height & Vo $_{2}$ max \\
\hline Placebo & $1.7 \pm 26.7$ & $16.8 \pm 79.2$ & $6.04 \pm 177.1$ & $11.1 \pm 49.6$ \\
\hline supplement & $2.7 \pm 25.2$ & $10.5 \pm 82.2$ & $6.5 \pm 178.9$ & $4.48 \pm 48.1$ \\
\hline \multicolumn{4}{|c}{ Scores are expressed as Mean \pm SD. } \\
\hline
\end{tabular}

Descriptive information about other research variables such as leukocytes, neutrophils, monocytes and lymphocytes, in four stages of pre-test, immediately after exercise, 1 hour after exercise and 2.5 hours after exercise, placebo group in Table 2 and supplement group Table 3, together with the results of Tukey post hoc test, is reported for the indices that have a significance level of $\mathrm{p}<0.05$ in the one-way ANOVA test (ANOVA).

Table 2 Changes indicators of case study placebo group in pre-test and post-test

\begin{tabular}{|c|c|c|c|c|c|c|c|c|}
\hline \multirow[t]{2}{*}{ Training variable } & \multicolumn{2}{|c|}{ Placebo group } & \multicolumn{2}{|c|}{ ANOVA } & \multicolumn{4}{|c|}{ Intergroup differences in Tukey test } \\
\hline & groups & $\begin{array}{l}\text { Mean and } \\
\text { stand. deviation }\end{array}$ & $\mathrm{F}$ & $\mathrm{P}$ & $\begin{array}{l}\text { Pre } \\
\text { test }\end{array}$ & Immediately & 1 hour & $\begin{array}{l}2.5 \\
\text { hours }\end{array}$ \\
\hline \multirow{4}{*}{$\begin{array}{l}\text { Leukocytes } \\
\text { (Number in } \\
\text { microliters) }\end{array}$} & Pre test & $786 \pm 129.1$ & \multirow{4}{*}{6.12} & \multirow{4}{*}{0.002} & & 0.006 & 0.99 & 0.073 \\
\hline & Immediately & $1220 \pm 310.8$ & & & & & 0.01 & 0.74 \\
\hline & 1 hour & $809 \pm 216.6$ & & & & & & 0.11 \\
\hline & 2.5 hours & $1095 \pm 270.2$ & & & & & & \\
\hline \multirow{4}{*}{$\begin{array}{l}\text { Neutrophils } \\
\text { (Percentage per } \\
\text { microliter) }\end{array}$} & Pre test & $60.4 \pm 8.4$ & \multirow{4}{*}{10.6} & \multirow{4}{*}{0.0001} & & 0.02 & 0.36 & 0.16 \\
\hline & Immediately & $48.8 \pm 11.9$ & & & & & 0.00 & 0.00 \\
\hline & 1 hour & $66.9 \pm 4.1$ & & & & & & 0.97 \\
\hline & 2.5 hours & $68.7 \pm 8.7$ & & & & & & \\
\hline \multirow{4}{*}{$\begin{array}{l}\text { Monocytes } \\
\text { (Percentage per } \\
\text { microliter) }\end{array}$} & Pre test & $2 \pm 6.3$ & \multirow{4}{*}{6.43} & \multirow{4}{*}{0.001} & & 0.5 & 0.79 & 0.001 \\
\hline & Immediately & $8 \pm 9.2$ & & & & & 0.97 & 0.04 \\
\hline & 1 hour & $6 \pm 8.4$ & & & & & & 0.012 \\
\hline & 2.5 hours & $20 \pm 13.3$ & & & & & & \\
\hline \multirow{4}{*}{$\begin{array}{l}\text { Lymphocytes } \\
\text { (Percentage per } \\
\text { microliter) }\end{array}$} & Pre test & $27.9 \pm 9.3$ & \multirow{4}{*}{12.19} & \multirow{4}{*}{0.0001} & & 0.02 & 0.25 & 0.07 \\
\hline & Immediately & $49.5 \pm 12.1$ & & & & & 0.00 & 0.00 \\
\hline & 1 hour & $20.5 \pm 3.6$ & & & & & & 0.092 \\
\hline & 2.5 hours & $28 \pm 7.7$ & & & & & & \\
\hline
\end{tabular}


Table 3 Changes indicators of case study supplement group in pre-test and post-test

\begin{tabular}{|c|c|c|c|c|c|c|c|c|}
\hline \multirow{2}{*}{$\begin{array}{l}\text { Training } \\
\text { variable }\end{array}$} & \multicolumn{2}{|c|}{ Placebo group } & \multicolumn{2}{|c|}{ ANOVA } & \multicolumn{4}{|c|}{ Intergroup differences in Tukey test } \\
\hline & groups & $\begin{array}{l}\text { Mean and } \\
\text { standard } \\
\text { deviation }\end{array}$ & $\mathrm{F}$ & $\mathrm{P}$ & $\begin{array}{l}\text { Pre } \\
\text { test }\end{array}$ & Immediately & 1 hour & 2.5 hours \\
\hline \multirow{4}{*}{$\begin{array}{l}\text { Leukocytes } \\
\text { (Number } \\
\text { microliters) }\end{array}$} & Pre test & $785 \pm 134.6$ & \multirow[t]{4}{*}{1.23} & \multirow[t]{4}{*}{0.31} & & & & \\
\hline & Immediately & $1670 \pm 15.72$ & & & & & & \\
\hline & 1 hour & $1225 \pm 299.5$ & & & & & & \\
\hline & 2.5 hours & $1119 \pm 200.9$ & & & & & & \\
\hline \multirow{4}{*}{$\begin{array}{l}\text { Neutrophils } \\
\text { (Percentage per } \\
\text { microliter) }\end{array}$} & Pre test & $53.7 \pm 8.2$ & \multirow[t]{4}{*}{7.92} & \multirow[t]{4}{*}{0.0001} & & 0.58 & 0.97 & 0.008 \\
\hline & Immediately & $48.5 \pm 10.5$ & & & & & 0.33 & 0.00 \\
\hline & 1 hour & $55.5 \pm 10.5$ & & & & & & 0.03 \\
\hline & 2.5 hours & $67.6 \pm 6.5$ & & & & & & \\
\hline \multirow{4}{*}{$\begin{array}{l}\text { Monocytes } \\
\text { (Percentage per } \\
\text { microliter) }\end{array}$} & Pre test & $3 \pm 4.8$ & \multirow[t]{4}{*}{2.9} & \multirow[t]{4}{*}{0.049} & & 0.99 & 0.57 & 0.11 \\
\hline & Immediately & $2 \pm 4.2$ & & & & & 0.42 & 0.04 \\
\hline & 1 hour & $8 \pm 7.9$ & & & & & & 0.72 \\
\hline & 2.5 hours & $12 \pm 13.9$ & & & & & & \\
\hline \multirow{4}{*}{$\begin{array}{l}\text { Lymphocytes } \\
\text { (Percentage per } \\
\text { microliter) }\end{array}$} & Pre test & $43.7 \pm 7.6$ & \multirow[t]{4}{*}{7.7} & \multirow[t]{4}{*}{0.0001} & & 0.46 & 0.96 & 0.01 \\
\hline & Immediately & $49.9 \pm 10.3$ & & & & & 0.0001 & 0.0001 \\
\hline & 1 hour & $41.7 \pm 11.8$ & & & & & & 0.04 \\
\hline & 2.5 hours & $30.2 \pm 6.9$ & & & & & & \\
\hline
\end{tabular}

\section{Discussion}

The results showed that in the glutamine group, there was no significant change in the number of wrestlers' blood leukocytes during the post-test compared to the pre-test.

While the results of research [18-21] indicate an increase in the number of leukocytes after taking glutamine supplementation. The difference in the results is due to the type of training and duration of activity, gender and level of readiness of the subjects. In the case of neutrophils, it increased significantly in the supplement group 2.5 hours after supplementation compared to immediately after exercise and 1 hour after supplementation. Comparing these results with the placebo group, it was observed that the increase in neutrophils at 1 and 2.5 hours after supplementation was less than the placebo group. Neutrophils produce lymphocytes that cause lymphocytes to reach below basal levels. Because glutamine acts as an inhibitor of prostaglandins as well as an antioxidant and fuel for immune cells, these changes can be attributed to glutamine supplementation by the experimental group. This result is consistent with the results of Mary Moore, et al [22], Jann Harberts, et al [21]. While Banafsheh [18] reported that after taking glutamine supplementation, the percentage of neutrophils in the supplement group was significantly reduced compared to the control group. The reason for the discrepancy between the results can be attributed to the differences in the sex of the subjects, the duration of training and also the time of supplementation in this study. Also, according to the research findings, the percentage of wrestlers' blood lymphocytes in both groups increased immediately after training compared to the pre-test, but this increase was not significant in the complementary group. Also, the percentage of lymphocytes in the supplement group was 2.5 hours after supplementation compared to the three pre-test stages, immediately after exercise, 1 hour after supplementation and also in the one-hour phase after supplementation compared to Decreased significantly immediately after exercise. The mechanisms of exercise-induced changes in lymphocytes have not been fully elucidated. Researchers believe that delayed administration of cortisol significantly reduces blood lymphocytes in the recovery period so that cortisol Cells prevent tissue from entering the bloodstream. Increased prostaglandins also cause lymphocytes to fall below basal levels. Reducing them during the recovery period and lowering them than the pre-workout level suppresses the immune system and thus increases the risk of upper respiratory tract infections in athletes. Compared with the placebo group, it was observed that the percentage of lymphocytes in 1 hour after supplementation decreased less than immediately after exercise in the supplement group compared to the placebo 
group. Due to the fact that glutamine acts as an inhibitor of prostaglandins and is widely used by lymphocytes, there can be less reduction in lymphocytes in the complement group than in the placebo group at different stages Attributed to glutamine intake. The result is consistent with the results of Mary Moore [22], Banafsheh [18], KURU et al [23]. While the result of de Siqueira research [24] showed an increase in the number of lymphocytes after taking glutamine supplementation. The reason for the discrepancy with the results of the present study can be attributed to the difference in training intensity and duration of activity. According to the results of the study, the percentage of monocytes in the blood of subjects in the supplement group increased significantly 2.5 hours after supplementation compared to immediately after exercise. At the same time, the monocytes in the placebo group also increased significantly, but the increase in the complement group was less than the placebo group. Researchers believe that the reason for the increase in monocytes after intense activity is due to the application of cortisol, the increase in monocytes is itself a sign of an infection. On the other hand, an increase in monocytes in the blood increases the level of prostaglandins released from these cells during 1.5 to 2 hours after strenuous exercise, which in turn reduces circulating lymphocytes and inhibits NK cells function. This result is consistent with de Siqueira's research [24]. While the results of Banafsheh [18] and Jann Harberts, et al [21] showed that glutamine supplementation cannot cause a significant change in the number of monocytes. The reason for the difference with the obtained results is due to the type and duration of training, gender and level of readiness of the subjects. KURU [24] also reported an increase in monocytes during a study in 2021. The discrepancy appears to be due to differences in the duration of supplementation. Ahmadi [19] reported that Bruce test could not make a significant difference in the number of monocytes. The reason for the difference with the obtained result is the difference in the level of readiness of the subjects. In general, because glutamine acts as an inhibitor of prostaglandins as well as an antioxidant and fuel for immune cells, any change in plasma glutamine concentration indirectly affects immune function. So it is possible that glutamine supplementation will moderate these changes to some extent.

One of the limitations of the present study is that if the study was conducted in a wider period of time and with a larger number of samples, it would be possible to comment on the results with more certainty. Overall, the evidence from this study shows that glutamine prevents certain immune system variables from decreasing or increasing too much, and reduces the detrimental effects of exercise to some extent.

\section{Conclusion}

The results of this study showed that glutamine consumption has beneficial effects on the immune system of athletes and prevents the weakening and suppression of the immune system due to strenuous activity. The available evidence is not strong enough to recommend the use of glutamine supplementation for the athlete, and further research is needed to elucidate the mechanisms of oral glutamine supplementation on immune system factors.

\section{Compliance with ethical standards}

\section{Acknowledgments}

Thanks to all the athletes who helped us with this research.

\section{Disclosure of conflict of interest}

The authors declare no conflict of interest in the present study.

\section{Statement of informed consent}

Informed consent was obtained from all individual participants included in the study.

\section{References}

[1] Shuqin Liu, Guoping Li, Junjie Xiao, Jing Wang. Exercise Regulates the Immune System. Physical Exercise for Human Health. 2020; 395-408.

[2] Ciro Alexandre Mercês Gonçalves, et al. Effect of Acute and Chronic Aerobic Exercise on Immunological Markers: A Systematic Review. Front. Physiol., 24 January 2020, https://doi.org/10.3389/fphys.2019.01602.

[3] Raisa de Oliveira Santos, Geovane da Silva Cardoso, Lara da Costa Lima, Mickael Laudrup de Sousa Cavalcante, Mariana Sousa Silva. l-Glutamine and Physical Exercise Prevent Intestinal Inflammation and Oxidative Stress Without Improving Gastric Dysmotility in Rats with Ulcerative Colitis. 2020; 44: 617-632. 
[4] Cruzat V, M Macedo Rogero, K Noel Keane, R Curi, P Newsholme. Glutamine: metabolism and immune function, supplementation and clinical translation. Nutrients. 2018; 10(11): 15-64.

[5] Ali Mujtaba Shah, Zhisheng Wang, Jian Ma. Glutamine Metabolism and Its Role in Immunity, a Comprehensive Review. Animals. 2021; 11(3): 905.

[6] Samjhana Thapaliya, Brian Gastman, Yee Peng Phoon, Chuan Li, Beiyan Zhou, Pauline Funchain. Role of glutamine metabolism in CD8+PD-1+TIM-3+ T cells in ICI resistant melanoma, Journal of clinical oncology. Volume 38, Issue 15, 2020.

[7] Leah Cooper, Ronald O Ball. Dispensable Amino Acids, except Glutamine and Proline, Are Ideal Nitrogen Sources for Protein Synthesis in the Presence of Adequate Indispensable Amino Acids in Adult Men. The Journal of Nutrition. September 2020; 150(9): 2398-2404.

[8] Wataru Aoi, Yuji Naito. Immune Function, Nutrition, and Exercise. Muscle Building, Endurance, and Strength. 2019; 83-95.

[9] Rainer H Straub. The brain and immune system prompt energy shortage in chronic inflammation and ageing. Nature Reviews Rheumatology. 2017; 13: 743-751.

[10] Vinicius Cruzat, Marcelo Macedo Rogero. Glutamine: Metabolism and Immune Function, Supplementation and Clinical Translation. Nutrients. 2018; 10(11): 1564.

[11] Sachin Kumar, Madhu Dikshit. Metabolic Insight of Neutrophils in Health and Disease. Front. Immunol. 10: 2099, 2019.

[12] Chen Zheng, Xiang-Ke Chen, Yue Zhou. Acute glutamine ingestion modulates lymphocytic responses to exhaustive exercise in the heat. Applied Physiology, Nutrition, and Metabolism. 43(3):213-220, 2017.

[13] Sandro Massao Hirabara et al. Host cell glutamine metabolism as a potential antiviral target. Clin Sci (Lond). 2021; 135(2): 305-325.

[14] Chirag HPatel, Jonathan DPowell. Targeting T cell metabolism to regulate $\mathrm{T}$ cell activation, differentiation and function in disease. Current Opinion in Immunology. June 2017; 46: 82-88.

[15] Shiv Bharadwaj, Mahendra Singh, Nikhil Kirtipal, Sang Gu Kang. SARS-CoV-2 and Glutamine: SARS-CoV-2 Triggered Pathogenesis via Metabolic Reprograming of Glutamine in Host Cells. Front. Mol. Biosci. 11 January 2021.

[16] Raquel Raizel, Julio Tirapegui . Role of glutamine, as free or dipeptide form, on muscle recovery from resistance training: a review study. Nutrire. 2018; 43: 28.

[17] Audrey Yule Coqueiro, et al. Effects of Glutamine and Alanine Supplementation on Central Fatigue Markers in Rats Submitted to Resistance Training. Nutrients. 2018, 10(2), 119.

[18] Banafsheh Amin, Mohammad Karimi. The Effect of Glutamine and Glucose Supplementation on Maximal Aerobic Power and Hemoglobin Concentration in Athlete Young Girls. Ann Appl Sport Sci. 2017; 5(1): 25-30.

[19] Amirhossein Ramezani Ahmadi, Elham Rayyani. The effect of glutamine supplementation on athletic performance, body composition, and immune function: A systematic review and a meta-analysis of clinical trials. Clinical Nutrition. June 2019; 38(3): 1076-1091.

[20] Aline CC Tritto, et al. Effect of rapid weight loss and glutamine supplementation on immunosuppression of combat athletes: a double-blind, placebo-controlled study. J Exerc Rehabil. 2018 Feb; 14(1): 83-92.

[21] Jann Harberts, et al. A Temperature-Controlled Patch Clamp Platform Demonstrated on Jurkat T Lymphocytes and Human Induced Pluripotent Stem Cell-Derived Neurons. Bioengineering. 2020; 7(2): 46.

[22] Mary Moore, et al. Oral Glutamine Supplement Reduces Subjective Fatigue Ratings during Repeated Bouts of Firefighting Simulations. Safety. 2019; 5(2): 38.

[23] Zehra Kuru, Mehmet Akman. Determination of Use Status of Supplements in Individuals Who Do Sports. International Journal of Health Administration and Education Congress (Sanitas Magisterium). 2021; 7(1): 93 105.

[24] De Siqueira R. Farinelli. Glutamine supplementation affects Th1 and Th2 cell populations in endurance horses. Comparative Exercise Physiology. 1 July 2020; 16(4): 259-266(8). 